\title{
HERRAMIENTAS GOOGLE EN EL APRENDIZAJE DE MATEMÁTICA FINANCIERA EN LOS ESTUDIANTES UNIVERSITARIOS
}

\author{
Abrahán Cesar Neri Ayala* \\ https://orcid.org/0000-0003-2799-3244 \\ Santiago Ernesto Ramos y Yovera** \\ https://orcid.org/0000-0001-9765-215X \\ Félix Gil Caro Soto*** \\ https://orcid.org/0000-0002-0946-5395
}

RECIBIDO: Diciembre 2019 / ACEPTADO: Abril 2020 / PUBLICADO: Mayo 2020

Como citar: Neri Ayala, Abrahán; Ramos y Yovera, Santiago; Caro Soto, Felix. (2020). Herramientas google en el aprendizaje de matemática financiera en los estudiantes universitarios. Telos: revista de Estudios Interdisciplinarios en Ciencias Sociales, 22 (2), Venezuela. (Pp.429-444).

DOI: www.doi.org/10.36390/telos222.13

\section{RESUMEN}

El presente artículo tuvo como objetivo determinar el efecto que causa el uso de Herramientas Google en el aprendizaje de matemática financiera en los alumnos del IV ciclo de la Escuela Profesional de Administración de la Universidad José Faustino Sánchez Carrión, Perú, usando como autores principales a: Suárez Sánchez-Ocaña (2012), Rojas (2014) Marmolejos et al (2014), Centorbi y Antonovich (2017), Castillo (2012), Otero et al (2019), Camargo y Hederich (2010), Díaz Barriga y Hernández (2010), Céspedes y Cossio (2015) y Hernández et al (2018). La misma se presenta por medio de un enfoque cuantitativo con diseño experimental. Este diseño, incorpora la aplicación de pre-pruebas y post-pruebas de los grupos que componen el experimento. Para la toma de datos se empleó una prueba de conocimiento sobre el aprendizaje de la matemática financiera, el diseño metodológico estuvo basado en la creación de un grupo control y uno experimental, cada grupo estuvo conformado por 23 estudiantes. Para comparar el promedio de los aprendizajes de los dos grupos se implementó el método estadístico no paramétrico $\mathrm{U}$ de Mann-Whitney obteniendo a través de un post test la cantidad de alumnos que exhibieron condiciones significativas dispares referenciadas a la prueba no paramétrica $U$ de Mann-Whitney $(p=0.000)$. Los alumnos del grupo experimental lograron mejores resultados (Promedio $=15,04$ ) luego del uso de las Herramientas Google, respecto a los alumnos del grupo control (Promedio $=10,52$ ). Se verificó que existen evidencias estadísticamente significativas para concluir que el uso de Herramientas Google mejora el aprendizaje de matemática financiera en los alumnos.

\footnotetext{
* Doctor en Educación. Universidad Nacional José Faustino Sánchez Carrión. Perú. Correo electrónico: neritoabrahan@gmail.com

** Doctor en Administración. Universidad Nacional José Faustino Sánchez Carrión. Perú. Correo electrónico: Ramusery1@gmail.com *** Doctor en Administración. Universidad Nacional José Faustino Sánchez Carrión. Perú. Correo electrónico: felixgilcarosoto@gmail.com
} 
Palabras claves: Herramientas Google, Aprendizaje, Matemática Financiera.

\title{
Google tools and the learning of financial mathematics in university students
}

\begin{abstract}
The objective of this article was to determine the effect of the use of Google Tools in the learning of financial mathematics in the students of the IV cycle of the Professional School of Administration of the University José Faustino Sánchez Carrión, Peru, using as principal authors a: Suárez Sánchez-Ocaña (2012), Rojas (2014) Marmolejos et al (2014), Centorbi y Antonovich (2017), Castillo (2012), Otero et al (2019), Camargo y Hederich (2010), Díaz Barriga y Hernández (2010), Céspedes y Cossio (2015) y Hernández et al (2018). It is presented through a quantitative approach with experimental design. This design incorporates the application of pretests and post-tests of the groups that make up the experiment. For the collection of data, a test of knowledge about learning financial mathematics was used, the methodological design was based on the creation of a control group and an experimental group, each group consisted of 23 students. To compare the average learning of the two groups, the non-parametric Mann-Whitney $\mathrm{U}$ statistical method was implemented, obtaining through a post-test the number of students who exhibited significant disparate conditions referenced to the non-parametric Mann-Whitney $U$ test $(p=0.000)$. The students in the experimental group achieved better results (Average $=15.04)$ after using Google Tools, compared to the students in the control group (Average $=10.52$ ). It was verified that there is statistically significant evidence to conclude that the use of Google Tools improves students' learning of financial mathematics.
\end{abstract}

Key words: Google Tools, Learning, Financial Mathematics.

\section{Introducción}

La idea de usar el nombre de Google se originó a través del término matemático "googol", o gogol en francés, que simboliza 10.100, es decir, un número que empieza con un 1 seguido de cien ceros). Google como empresa fue fundada en septiembre de 1998 desarrollando como producto bandera el buscador de internet introducido por Larry Page y Sergey Brin (Suárez Sánchez-Ocaña, 2012).

Además, de posicionarse como el buscador más empleado en el mundo, este cuenta con herramientas que está a disposición de la comunidad educativa internacional, permitiendo de esta manera que en el área educativa muchos docentes y estudiantes la utilicen como herramienta en su labor pedagógica y de aprendizaje (Essay, 2018).

Según la Organización de las Naciones Unidas para la Educación, la Ciencia y la Cultura (UNESCO, 2015),, la educación se posiciona en un puesto notorio en la ruta de los avances educativos a nivel mundial, formando parte de sus objetivos para el desarrollo sostenible de la educación a través del impulso de nuevas estrategias de enseñanza enfocados 
al progreso del mundo reconociendo así el poder que tiene la educación en los diferentes niveles educativos y sociales como un aspecto fundamental para su desarrollo.

Basados en el poder que tiene la educación para el desarrollo de una nación, Rojas (2014) expresa que la matemática financiera simboliza un diverso grupo de saberes numéricos que están asociados a la economía que a lo largo de la historia han consolidado las estructuras de dominación reinantes en el común de los países del mundo. Debido a su importancia se considera imperioso realizar un análisis de la manera con que tradicionalmente se ha enfocado la enseñanza través del tiempo bajo contextos educativos distorsionantes y pocos eficientes. Según Marmolejos et al., (2014), el mundo del conocimiento demanda de expertos preparados en ciencias naturales y matemáticas, con capacidad para solucionar problemas e ideas que permitan tomar decisiones; preparados para discernir, transformar y generar diversos mensajes; que permita ampliar el pensamiento abstracto, y tener la seguridad en el momento que se esté en la búsqueda de soluciones teniendo un criterio crítico que permita generar cambios científicotecnológicos.

Es por ello que, la matemática financiera se aplica durante los cálculos de diferentes tipos de crédito los intereses generados y anualidades a pagar, de igual manera, permite además investigar todos los factores que actúan a través del tiempo en el cambio del valor monetario, por lo que su estudio es básico para las negociaciones crediticias que a través del tiempo ha generado un sin fin de procedimientos financieros ejecutados en la economía de una nación (Centorbi y Antonovich, 2017).

Así mismo, debido a que la enseñanza es considerada un proceso eficiente que permite la creación de docentes con nuevas concepciones, estos deben estar enmarcados en la generación de innovadores puntos de vista, con una capacidad de entendimiento y enseñanza basados en la flexibilidad para promover el trabajo en equipo, fundamentados en esas nuevas tecnologías; esto con el fin de indagar sobre el perfeccionamiento de ese conocimiento; permitiendo además la inclusión consciente del estudiante a una sociedad basada en reglas, conductas, instrucciones, valores, entre otros, en coherencia con el medio cultural en el que se desenvuelve el alumnado (Castillo, 2012).

En ese sentido, Otero et al. (2019), plantean en los resultados de su estudio que en la actualidad que el conjunto de herramientas presentes en las páginas Web y las Apps

Conllevan de manera ineluctable nuevos cambios sociales propios de la "Plantean en los resultados de su estudio, que en la actualidad que las aplicaciones utilizables en la Web y los AppStore conllevan de manera ineluctable nuevos cambios sociales propios de la globalización, ciertos docentes se sienten inmigrantes digitales y enfrentan el desafío de introducirse en el entorno digital en el cual los estudiantes ya están inmersos; en la mayoría de los casos, los docentes son receptivos frente al uso de estas tecnologías siempre y cuando sean involucrados en los procesos de apropiación y encuentren los mecanismos a través de los cuales sus contenidos y metodologías se articulen con el uso de nuevas tecnologías (p.537).

De este modo, los avances tecnológicos se han apoderado de las aulas de la Universidad Nacional José Faustino Sánchez Carrión (Perú), siendo los Smartphone los más utilizados por los estudiantes, y las Tablets por parte de los docentes para dictar sus clases; observándose, 
además el uso de las aulas virtuales como redes de comunicación para que los estudiantes puedan completar sus deberes, comunicarse con sus compañeros y profesores de forma remota.

El presente estudio sobre herramientas Google y el aprendizaje de matemática financiera en los estudiantes de la Universidad en estudio, tiene como propósito especificar el resultado que se genera con la puesta en práctica de las herramientas Google durante el proceso de aprendizaje de matemática financiera, con el mismo pretende además deducir y entender la importancia del uso de ese tipo de herramientas en el aprendizaje de la matemática. Para lo cual es necesario generar un conocimiento teórico y práctico fortalecido que les permita generar un proceso de aprendizaje más fortalecido, y así, enriquecer el proceso de enseñanza- aprendizaje impartido en las asignaturas que se dicta en esta casa superior de estudios.

\section{Marco teórico}

\section{Herramientas de Google}

Las herramientas de google son un conglomerado de aplicaciones que facilita la organización del trabajo de forma sencilla, así como el intercambio entre los diferentes usuarios con múltiples herramientas para la generación del servicio de correo electrónico, programación de reuniones y trabajos a través de la mensajería instantánea, la creación de hojas de cálculo, y documentos (Avello y Duart, 2016).

La constante disposición de nuevos recursos tecnológicos favorece la creación de innovaciones, creando diferentes usos para el beneficio de los estudiantes; por lo que este grupo de herramientas han ayudado a que a nivel educativo se genere un proceso de innovación digital superando así los antiguos procesos de enseñanza y aprendizaje aplicados a nivel universitario (Garay, 2012), generando de alguna manera la superación de obstáculos y limitaciones geográficas que antes existían, esto gracias a la invención de aplicaciones educativas para perfeccionar el proceso de enseñanza aprendizaje; entre ese grupo de nuevas aplicaciones se encuentran classroom, formularios, drive, calendar, hangounts, entre otras, (Iftakhar, Shampa, 2016).

\section{Google Drive}

El Google Drive está referido al servicio de almacenaje de archivos gratuitos en línea, asimismo se pueden crear nuevos documentos, editar y compartir los almacenados como hojas de cálculo documentos, presentación entre otro tipo de archivos (Barrios y Casadei Carniel, 2014).

\section{Google Classroom}

Google Classroom presenta un acceso gratuito para la comunidad universitaria a través de la aplicación Google Apps for Education (GAE), desarrollada por Google, y generada con el fin de administrar otras aplicaciones como Google Documents, Google Drive, Google Forms y Google Calendar; este grupo de aplicaciones permiten la instauración de un aula virtual a través del cual se pueda generar pruebas para la distribución de lecturas, videos, tareas, entre otras funciones (Vélez, 2016).

Con esta herramienta, el docente fija una labor a ejecutar en conjunto a un documento almacenado en Google Drive; luego los estudiantes recogen una copia de ese documento, incluidas las instrucciones de la asignación, trabajan sobre el documento para realizar la labor 
asignada y lo envían al profesor. Posteriormente el docente corrige ese documento para cada estudiante, lo califica y se lo regresa con las correcciones respectivas.

\section{Google Hangounts}

Según Borras y Pérez (2017), por medio de esta herramienta de puede realizar videoconferencias, en las cuales los participantes expresan destrezas aprendidas y otros conocimientos, que permita inducir el intercambio de conocimientos entre los involucrados. Fomentar estas herramientas en ambientes con alto número de colaboradores a través de las clases masivas abiertas en línea, generará el desarrollo de la inteligencia del grupo de estudio, siendo por lo tanto un recurso de enseñanza aprendizaje muy novedoso pues siempre está enmarcado en la presencia de actualizaciones en sus formatos.

\section{Google Formulario}

La creación de formularios por parte de Google, como Google Forms no tiene una definición como tal, más bien se pueden encontrar breves descripciones de lo que se puede hacer por medio de esa aplicación; recomendándose la planificación de eventos, envío de encuestas, planteamientos de preguntas a cierto público o compilar una gran diversidad de información de manera fácil y eficiente, según su propia descripción, la cual, se considera, refleja parcialmente la verdadera gama de usos que ofrece Leyva et al., (2018).

Por lo que se puede decir que este tipo de formularios, son un recurso de fácil uso excepcionalmente utilizado por su simplicidad de uso y por el acceso al mismo por una red de internet, y rapidez por medio de una búsqueda específica de los resultados. A través de estos formularios, se crean documentos de texto y recursos multimedia como imágenes, sonido y vídeo, lo que amplía las oportunidades de uso como herramienta de evaluación, así como la oportunidad de mostrar una diversidad de configuraciones y presencia de una variedad de gráficas (Valijas de herramientas TIC, 2016).

\section{Aprendizaje de matemática financiera}

La Matemática, como sistema de saberes establecidos en permanente expansión, es empleada en la mayoría de las disciplinas de los saberes y primordialmente en las ciencias fiscales (Durán y Durán, 2018), esta permite ajustar la realidad y emplear el sentido lógico para abordar a generalidades, empleando la categorización. Aching, (2005), la considera como una división de la matemática aplicada que tiene como fin analizar la variación a través del tiempo del dinero, a través del análisis del capital, las tasas y el tiempo que se necesita para concebir ganancias, todo esto con el uso de técnicas de evaluación que generen nuevas y mejores medidas al momento de invertir, de igual manera se conoce como análisis de inversiones, administración de inversiones o ingeniería económica.

La Matemática es considerada además como una de las asignaturas que beneficia el análisis económico y financiero de una nación; por esto se considerada como uno de los principales objetivos planteados en todo currículo, debido a que esta genera en los individuos un mejor entendimiento, de su entorno y su interrelación con este (Castro, 2007).

De igual manera, está asociada con la contabilidad en el uso de información racional obtenida de los registros contables; siendo una herramienta muy útil complementaria además de la ciencia política, esto debido a su uso en el estudio y solución de problemas económicos 
directamente relacionados con la sociedad, lo que ayuda a esta rama al momento de tomar de decisiones de presupuesto, inversión y ajustes económicos (Pérez et al., 2018). Por lo que se puede considerar que la matemática financiera tiene implementación práctica, y su estudio es directamente proporcional a la solución de situaciones cotidianas en múltiples áreas.

\section{Aprendizaje conceptual}

El aprendizaje conceptual es aquel donde el alumno transforma estructuras lógicas mentales (relaciones y conceptos) en relación al análisis o proceso de la información afín con el objeto de estudio que desea aprender (González et al., 2018).

Por medio de este tipo de aprendizaje el usuario elabora estructuras lógicas mentales como conceptos y relaciones a través del estudio 0 análisis de la información asociada con el objeto que desea aprender (Camargo y Hederich, 2010).

Según Montilva y Montilva (2018), es considerado un proceso de conceptualización o formación de conceptos que abarca la simbolización de los conceptos esenciales en una tecnología, estableciendo relaciones entre los conceptos identificados y de las propiedades de cada concepto.

\section{Aprendizaje procedimental}

El conocimiento procedimental, o conocimiento de cómo realizar actividades cognoscitivas es fundamental para gran parte del aprendizaje escolar (Montilva y Montilva, 2018). Este conocimiento se usa además para solucionar dificultades matemáticas, sintetizar información, inspeccionar párrafos velozmente y emplear técnicas de laboratorio.

Por otra parte, el conocimiento procedimental se almacena como imágenes o códigos verbales, de forma parecida a como se adquiere el conocimiento declarativo. La teoría del control adaptativo del comportamiento, propone que el conocimiento procedimental se adquiere como un sistema de producción (Díaz y Hernández, 2010)

\section{Aprendizaje Actitudinal}

Céspedes y Cossio (2015), expresan que los contenidos actitudinales se refieren a la diversidad de maneras de cómo se pueden presentar las realidades de las acciones humanas, a partir de la particularidad que le genera cada personas o situaciones o hechos, dependiendo de cómo el individuo se relacione con el proceso desde lo cognitivo, lo afectivo y lo conductual.

Con respecto a la actitud, son disposiciones aproximadamente invariables de los individuos a comportarse de cierta forma. Es la manera en que el individuo sintetiza su actitud según determinados valores (Chávez y Villacorta, 2019). Entonces, formarse según una actitud en específico involucra, actuar y sentir de modo más o menos invariable ante una entidad determinada a quien va dirigida la actitud.

\section{Metodología}

Este estudió fue experimental, con una operacionalización de variables de manera deliberada. Según Hernández et al. (2018), en las variables independientes (supuestas causas) se analizan la o las consecuencias producto de la manipulación respecto a la o las variables dependientes (supuestos efectos) bajo un escenario controlado. 
La variable manipulada corresponde a las Herramientas Google (variable independiente) para analizar las consecuencias en el aprendizaje de matemática financiera (variable dependiente), verificando la relación causa- efecto entre ambas variables. En ese sentido, el nivel de la presente investigación es explicativo con un diseño experimental con modelo cuasiexperimental.

La unidad de análisis estuvo conformada por 46 estudiantes provenientes del curso de matemática financiera de la escuela profesional de Administración de la Universidad Nacional José Faustino Sánchez Carrión, Perú. La investigación se realizó desde junio de 2017 a marzo de 2018; los grupos estudiados fueron homogéneos e intactos, por lo que la muestra seleccionada para la investigación fue un grupo de control con 23 y un grupo experimental con 23 estudiantes.

Se aplicó la prueba objetiva como instrumento para la toma de datos, la cual fue validada por grupo de expertos en el área, su validez fue verificada a través de la fórmula Alfa Conbrach, obteniendo un coeficiente de 0.96 (cercano a 1), lo cual indica que el instrumento tiene una alta confiabilidad.

Este diseño, estuvo basado además con la incorporación de pre-pruebas y post-pruebas a los grupos experimentales (Hernández et al., 2018). Los individuos estudiados fueron fijados al azar en los grupos, posteriormente se les aplicó de manera simultánea una pre-prueba (prueba de entrada), un grupo recibió el tratamiento experimental con las Herramientas Google, aplicadas para lograr el aprendizaje y el otro sin estas herramientas (grupo de control), en último lugar se les administró paralelamente una post-prueba (prueba de salida) (tabla 1).

Tabla 1. Esquema de experimentación aplicado.

\begin{tabular}{|c|c|c|c|c|c|}
\hline GRUPO & ASIGNACIÓN & $\begin{array}{c}\text { PRE } \\
\text { PRUEBA }\end{array}$ & TRATAMIENTO & $\begin{array}{c}\text { POST } \\
\text { PRUEBA }\end{array}$ & DIFERENCIA \\
\hline GE & $\mathrm{R}$ & 01 & $\mathrm{X}$ & 02 & $02-01=\mathrm{D} 1$ \\
\hline GC & - & 03 & - & 04 & $04-03=\mathrm{D} 2$ \\
\hline
\end{tabular}

Fuente: Elaboración propia

Dónde: Asignar al azar (R), Grupo de sujetos (G) (G.E: grupo experimental que recibe tratamiento, G:C: grupo control que no recibe tratamiento), Tratamiento, estímulo, reactivo experimental $(\mathrm{X})$, Medición realizada a uno o más grupos a través de pruebas, cuestionarios, observaciones, entre otros $(0)$, ausencia de tratamiento (-).

En resumen, se les aplicó las técnicas de observación, entrevista, encuesta y test de performance. Los datos fueron recolectados según los siguientes pasos:

1. Verificación de las pruebas objetivas de entrada (pre-test) y salida (post-test).

2. Pruebas piloto para realizar posteriores modificaciones.

3. Llenado de la ficha con información general.

4. Coordinación con el encargado de la dirección de estudios para el acceso al centro de cómputo y la aplicación de las Herramientas Google.

5. Ejecución de pruebas de entrada o el pre-test al grupo control y al grupo experimental. 
6. Aplicación de las herramientas Google al Grupo Experimental

7. Ejecución del post-test a los dos grupos.

8. Ingreso de los resultados alcanzados al software estadístico SPSS para el estudio estadístico.

9. Se realizó operaciones estadísticas para evidenciar si las hipótesis planteadas eran aceptadas 0 refutadas.

En consecuencia, surgen algunas interrogantes básicas, como: ¿Cuál es el efecto que genera el uso de herramientas Google en el aprendizaje de matemática financiera en los estudiantes? Por lo que, se propuso implementar su uso para mejorar los aprendizajes de matemática financiera en estudiantes de la escuela profesional de Administración de la Universidad en estudio. Y se formula la siguiente hipótesis de investigación: el uso de Herramientas Google mejora el aprendizaje de matemática financiera en los estudiantes del IV ciclo de la escuela profesional de Administración.

Para comparar el promedio de los aprendizajes de los dos grupos se implementó el método estadístico no paramétrico $U$ de Mann-Whitney el cual se aplica a dos muestras independientes, siendo la prueba no paramétrica de la habitual prueba t de Student y se usa para comprobar la heterogeneidad de dos muestras ordinales.

\section{Resultados}

Después de la aplicación de las herramientas en cuestión al grupo experimental en comparación con el grupo control se presentan los siguientes resultados.

El grupo control durante el pre-test estuvo representado por un $56,5 \%$ de los estudiantes en estudio demostrando un nivel deficiente en el aprendizaje de matemática financiera y un $43,5 \%$ con un nivel aceptable. Asimismo, en el post-test el $52,2 \%$ presentó un nivel deficiente y un $34,8 \%$ un nivel aceptable (tabla 2 ).

Tabla 2. Nivel de aprendizaje obtenido en matemática financiera

\begin{tabular}{cccccc}
\hline \multirow{2}{*}{ Grupo } & & \multicolumn{2}{c}{ Pre Test } & \multicolumn{2}{c}{ Post Test } \\
\cline { 3 - 6 } & Categoría & $\mathrm{f}$ & $\%$ & $\mathrm{f}$ & $\%$ \\
\hline \multirow{3}{*}{ Control } & Deficiente & 13 & $56,5 \%$ & 12 & $52,2 \%$ \\
& Aceptable & 10 & $43,5 \%$ & 8 & $34,8 \%$ \\
& Bueno & 0 & $0,0 \%$ & 3 & $13,0 \%$ \\
& Excelente & 0 & $0,0 \%$ & 0 & $0,0 \%$ \\
\cline { 2 - 6 } & Total & 23 & $100,00 \%$ & 23 & $100,00 \%$ \\
\hline \multirow{5}{*}{ Experimental } & Deficiente & 12 & $52,2 \%$ & 0 & $0,0 \%$ \\
& Aceptable & 11 & $47,8 \%$ & 6 & $26,1 \%$ \\
& Bueno & 0 & $0,0 \%$ & 11 & $47,8 \%$ \\
& Excelente & 0 & $0,0 \%$ & 6 & $26,1 \%$ \\
\hline
\end{tabular}

Fuente: Elaboración propia según SPSS.

En el caso del grupo experimental durante el pre-test el 52,2\% alcanzó un nivel deficiente en el aprendizaje de la matemática financiera y un $47,8 \%$ obtuvieron un nivel aceptable. En el 
caso del post-test un $47,8 \%$ presentó un nivel bueno, el $26,1 \%$ presentó un nivel aceptable y un $26,1 \%$ un nivel excelente.

En la tabla 3 se aprecia como el grupo control, durante el pre-test un 47,8\% alcanzó un nivel deficiente en el aprendizaje conceptual de la matemática financiera, un $39,1 \%$ alcanzó un nivel aceptable y sólo un 13,0\% alcanzó un nivel bueno. En el caso del post-test el 39,1\% obtuvieron un nivel aceptable y un $34,8 \%$ un nivel bueno.

Tabla 3. Nivel de aprendizaje conceptual obtenido en matemática financiera

\begin{tabular}{cccccc}
\hline \multirow{2}{*}{ Grupo } & & \multicolumn{2}{c}{ Pre Test } & \multicolumn{2}{c}{ Post Test } \\
\cline { 2 - 6 } & Categoría & $f$ & $\%$ & $f$ & $\%$ \\
\hline \multirow{3}{*}{ Control } & Deficiente & 11 & $47,8 \%$ & 6 & $26,1 \%$ \\
& Aceptable & 9 & $39,1 \%$ & 9 & $39,1 \%$ \\
& Bueno & 3 & $13,0 \%$ & 8 & $34,8 \%$ \\
& Excelente & 0 & $0,0 \%$ & 0 & $0,0 \%$ \\
\cline { 2 - 6 } & Total & 23 & $100,00 \%$ & 23 & $100,00 \%$ \\
\hline \multirow{2}{*}{ Experimental } & Deficiente & 10 & $43,5 \%$ & 0 & $0,0 \%$ \\
& Aceptable & 10 & $43,5 \%$ & 2 & $8,7 \%$ \\
& Bueno & 3 & $13,0 \%$ & 11 & $47,8 \%$ \\
& Excelente & 0 & $0,0 \%$ & 10 & $43,5 \%$ \\
\hline
\end{tabular}

Fuente: Elaboración propia según SPSS.

Según el enfoque de aprendizaje conceptual el grupo de experimental según el pre-test un $43,5 \%$ alcanzó un nivel deficiente en el aprendizaje conceptual de la matemática financiera y un $43,5 \%$ obtuvieron un nivel aceptable. En el caso del Post test el 47,8\% se encontró en un nivel bueno de aprendizaje, un $43,5 \%$ un nivel excelente y un $8,7 \%$ un nivel aceptable.

En la tabla 4 se concretan los porcentajes del grupo control, en el caso del pre-test un $52,2 \%$ alcanzó un nivel deficiente en el aprendizaje procedimental de la matemática financiera y un $43,5 \%$ obtuvieron un nivel aceptable. Y en el post-test el $47,8 \%$ obtuvieron un nivel deficiente y un $30,4 \%$ un nivel aceptable.

Tabla 4. Nivel de aprendizaje procedimental obtenido en matemática financiera

\begin{tabular}{cccccc}
\hline \multirow{2}{*}{ Grupo } & & \multicolumn{3}{c}{ Pre Test } & \multicolumn{2}{c}{ Post Test } \\
\cline { 2 - 5 } & Categoría & $\mathrm{f}$ & $\%$ & $\mathrm{f}$ & $\%$ \\
\hline \multirow{3}{*}{ Control } & Deficiente & 12 & $52,2 \%$ & 11 & $47,8 \%$ \\
& Aceptable & 10 & $43,5 \%$ & 7 & $30,4 \%$ \\
& Bueno & 1 & $4,3 \%$ & 4 & $17,4 \%$ \\
& Excelente & 0 & $0,0 \%$ & 1 & $4,3 \%$ \\
\hline \multirow{2}{*}{ Experimental } & Total & 23 & $100,00 \%$ & 23 & $100,00 \%$ \\
\hline \multirow{2}{*}{} & Deficiente & 9 & $39,1 \%$ & 3 & $13,0 \%$ \\
& Aceptable & 9 & $39,1 \%$ & 4 & $17,4 \%$ \\
& Bueno & 5 & $21,7 \%$ & 6 & $26,1 \%$ \\
& Excelente & 0 & $0,0 \%$ & 10 & $43,5 \%$
\end{tabular}




\begin{tabular}{ccccc}
\hline Total & 23 & $100,00 \%$ & 23 & $100,00 \%$ \\
\hline
\end{tabular}

Fuente: Elaboración propia según SPSS.

Según el enfoque de un aprendizaje procedimental de matemática financiera el grupo experimental según el Pre test el $39,1 \%$ presentó un nivel deficiente de aprendizaje y un $39,1 \%$ obtuvo un nivel aceptable. En el caso del Post test el $43,5 \%$ obtuvieron un nivel excelente, un $26,1 \%$ un nivel bueno y un $17,4 \%$ un nivel aceptable.

En la tabla 5 se detallan los porcentajes del grupo control, en el pre-test el 78,3\%, obteniéndose un nivel deficiente en el aprendizaje actitudinal de la matemática financiera y un $21,7 \%$ obtuvieron un nivel aceptable. $Y$ en el post-test el $65,2 \%$ obtuvieron un nivel deficiente y un $26,1 \%$ un nivel aceptable.

Tabla 5. Nivel de aprendizaje actitudinal obtenido en matemática financiera.

\begin{tabular}{cccccc}
\hline \multirow{2}{*}{ Grupo } & & \multicolumn{2}{c}{ Pre Test } & \multicolumn{2}{c}{ Post Test } \\
\cline { 3 - 6 } & Categoría & $\mathrm{f}$ & $\%$ & $\mathrm{f}$ & $\%$ \\
\hline \multirow{4}{*}{ Control } & Deficiente & 18 & $78,3 \%$ & 15 & $65,2 \%$ \\
& Aceptable & 5 & $21,7 \%$ & 6 & $26,1 \%$ \\
& Bueno & 0 & $0,0 \%$ & 0 & $0,0 \%$ \\
& Excelente & 0 & $0,0 \%$ & 2 & $8,7 \%$ \\
\cline { 2 - 6 } & Total & 23 & $100,00 \%$ & 23 & $100,00 \%$ \\
\hline \multirow{3}{*}{ Experimental } & Deficiente & 16 & $69,6 \%$ & 0 & $0,0 \%$ \\
& Aceptable & 7 & $30,4 \%$ & 11 & $47,8 \%$ \\
& Bueno & 0 & $0,0 \%$ & 0 & $0,0 \%$ \\
& Excelente & 0 & $0,0 \%$ & 12 & $52,2 \%$ \\
\hline
\end{tabular}

Fuente: Elaboración propia según SPSS.

Por su parte, el grupo experimental según el enfoque de un aprendizaje actitudinal de matemática financiera para el pre-test alcanzó un $69,6 \%$ de nivel presentó un nivel excelente y un $47,8 \%$ un nivel aceptable de aprendizaje.

La prueba de normalidad demostró que los datos no presentaban una distribución normal, debido a que el valor de "p" contrastado con el valor de Shapiro - Wilk está por encima del nivel del valor $p<0,05$; en este caso se empleó la prueba no paramétrica test $U$ de Mann-Whitney (tabla 6).

Tabla 6. Test de normalidad de SHAPIRO -WILK

\begin{tabular}{lcccc}
\hline \hline \multirow{2}{*}{ Variables y dimensiones } & \multirow{2}{*}{ Grupo } & \multicolumn{3}{c}{ Shapiro-Wilk } \\
\cline { 3 - 5 } & & Estadístico & gl & Sig. \\
\hline \multirow{2}{*}{ Pre test Aprendizaje conceptual } & Control &, 881 & 23 &, 010 \\
\multirow{2}{*}{ Pre test Aprendizaje procedimental } & Experimental &, 797 & 23 &, 000 \\
\cline { 2 - 5 } & Control &, 835 & 23 &, 001
\end{tabular}




\begin{tabular}{lcccc} 
& Control &, 810 & 23 &, 001 \\
Pre test Aprendizaje actitudinal & Experimental &, 866 & 23 &, 005 \\
\cline { 2 - 5 } Pre test Aprendizaje de la & Control &, 906 & 23 &, 004 \\
matemática financiera & Experimental &, 912 & 23 &, 005 \\
\cline { 2 - 5 } Post test Aprendizaje conceptual & Control &, 871 & 23 &, 007 \\
Post test Aprendizaje & Experimental &, 831 & 23 &, 001 \\
\cline { 2 - 5 } procedimental & Control &, 898 & 23 &, 003 \\
Post test Aprendizaje actitudinal & Experimental &, 816 & 23 &, 001 \\
\cline { 2 - 5 } & Control &, 855 & 23 &, 003 \\
Post test Aprendizaje de la & Experimental &, 639 & 23 &, 000 \\
matemática financiera & Control &, 952 & 23 &, 004 \\
\cline { 2 - 5 } & Experimental &, 912 & 23 &, 004 \\
\hline \hline
\end{tabular}

Fuente: Elaboración propia según SPSS.

En ese sentido, a través de la prueba $U$ de Mann-Whitney, se contrastó la hipótesis alternativa de que el uso de Herramientas Google mejora el aprendizaje de matemática financiera en los alumnos en estudio. Y la hipótesis nula de que el uso de Herramientas Google no mejora ese aprendizaje (tabla 7).

Tabla 7. El aprendizaje de matemática financiera en los estudiantes de la escuela profesional de Administración.

\begin{tabular}{|c|c|c|c|}
\hline \multirow[b]{2}{*}{ Estadístico } & \multicolumn{2}{|c|}{ Grupo } & \multirow{2}{*}{$\begin{array}{c}\text { Test U de } \\
\text { Mann- } \\
\text { Whitneya }\end{array}$} \\
\hline & Control $(n=23)$ & Experimental $(n=23)$ & \\
\hline \multicolumn{4}{|c|}{ Pre test } \\
\hline Media & 9,91 & 10,43 & $\mathrm{U}=217.000$ \\
\hline Desviación típica & 1,47 & 1,16 & $\begin{array}{l}Z=-1.0 / 3 \\
p=0.283\end{array}$ \\
\hline \multicolumn{4}{|c|}{ Post test } \\
\hline Media & 10,52 & 15,04 & $\mathrm{U}=37.000$ \\
\hline Desviación típica & 2,06 & 2,22 & $\begin{array}{c}Z=-5.033 \\
p=0.000\end{array}$ \\
\hline
\end{tabular}

Fuente: Elaboración propia según SPSS.

Según el U de Mann-Whitney para el pre test se observó con un 95\% de confiabilidad y un nivel de significancia del $5 \%$ que el aprendizaje en los alumnos de ambos grupos presentó al inicio condiciones parecidas $(p=0.2838)$ superior a 0.05 .

Sin embargo, para el post test los alumnos presentaron diferencias significativas con un $p=0.000$ para ambos grupos, siendo los alumnos del grupo experimental los que presentaron un mejor Promedio $=15,04$ después del uso de las Herramientas Google, comparados con los alumnos del grupo control quienes obtuvieron un Promedio $=10,52$. 
Los resultados obtenidos demuestran que el uso de Herramientas Google genera un avance el aprendizaje de matemática financiera en el grupo de estudio, por lo cual se acepta la hipótesis de investigación.

\section{Discusión de resultados}

En ese sentido, los resultados corroboran lo expresado por Valdés (2019), quien resalta este tipo de tecnología apoyan de gran manera a los educadores durante su capacitación y así estar a la par con los nuevos avances tecnológicos a nivel educativo que les permita estar en un mejor nivel profesional, lo que permite estimular su uso en sus diferentes actividades educativas; así como buscar herramientas que les permitan generar un mayor acceso a la información para compartir sus investigaciones con otros investigadores generando actividades curriculares más agiles y colaborativas.

De igual manera, se relacionan con los aportes de Otero et al. (2019), quienes en su estudio señalan que el uso de las TIC aporta significativamente a la evolución del estudiante; pasando de un estado pasivo a uno activo dentro del desarrollo de aprendizaje. Por lo que una vez más se afirma que permitir el acceso a este tipo de recurso genera un enriquecimiento de su conocimiento de manera colaborativa y participativa.

Generalmente en el área educativa las TICs son usadas para el desarrollo cognoscitivo de los alumnos, sin embrago el resultado de la encuesta aplicada reflejó que si estas son mal usadas por no existir una continua formación de los docentes que les permita obtener agilidad en su uso y el conocimiento de las continuas actualizaciones que presentan este tipo de herramientas; esto se pudo observar en la encuesta realizada donde estos manifestaron la ausencia de un manual de apoyo sobre dichas herramientas tecnológicas.

Además, los resultados son confirmados por los obtenidos por Roque (2017), el cual expone la relación significativa que existe entre el manejo de las TICs y el aprendizaje en la comunicación de los discentes, lo que muestra que las TICs posibilitan al educador originar un aprendizaje significativo en estos.

En forma similar, se amplían de forma significativa los aportes de Galantini (2015), quien soporta la teoría de la presencia de una correlación significativa entre la estimulación que se origina por el uso de la herramienta Google Site para foros virtuales.

Por otra parte, contradicen los planteamientos de Maldonado (2014), quien afirma que para la enseñanza de la Geografía por medio de este tipo de tecnología no genera ninguna mejora en los resultados del aprendizaje de los alumnos sin el acompañamiento de las herramientas tradicionales. Siendo esto por la simple razón, de que son medios de enseñanza usados como estrategias, los cuales permiten de igual manera una diversidad de metodologías y estrategias. Por lo que cualquier propuesta de cambio tecnológico necesitará del nivel de bienestar alcanzado en alumnos y docentes.

En términos generales se puede establecer que existe evidencia suficiente para concluir que el uso de Herramientas Google mejoró el aprendizaje de matemática financiera en el grupo de alumnos estudiados.

\section{Conclusiones}

Según los resultados observados permiten reconocer que el uso adecuado de las Herramientas de Google, fortalecen el proceso de aprendizaje en los alumnos a través de los 
diferentes tipos de aprendizajes como lo es el aprendizaje conceptual, pues en este caso se abarcó el hecho de que los estudiantes fuesen capaces de explorar y relacionar particularidades frecuentes, en sus aprendizajes de la matemática financiera, en el caso del aprendizaje actitudinal pues se tuvo como fin que el estudiante tenga capacidad reflexiva sobre su comportamiento ante este tipo de herramientas que mejoran su proceso de aprendizaje con nuevas y mejores comportamientos e ideas generando una actuación basado en la coherencia entre la actitud que poseía ante la matemática financiera aprendidas bajo este tipo de herramientas y otras que existen en el medio educativo y procedimental, puesto este tipo de aprendizaje se implementa con la idea que sirva de provecho para el incremento de las experiencias, mediante el ejercicio reflexivo en varias áreas para ejecutar acciones específicas.

Y, por último, se concluye, además que el uso de Herramientas Google mejora el aprendizaje procedimental de la matemática financiera en los estudiantes del IV estudiados esto es muy relevante, ya que se estaría fortaleciendo las acciones de manera positiva, ateniendo las apreciaciones de la humanidad en la que se vive, incentivando al estudiante a formar una idiosincrasia que anteponga un actuar idóneo ante cualquier circunstancia.

Se pudo conocer la presencia de algunas limitaciones en el proceso de Enseñanzaaprendizaje, debido a la poca interacción que los docentes y estudiantes tienen con la tecnología, por el poco uso laboratorio de computación que, a pesar de contar con internet, incumplían además con las exigencias del ministerio de educación relacionado el uso de avances tecnológicos.

Es importante reconocer que este tipo de tecnologías permiten al docente mejorar su nivel competitivo y laboral, por lo que es necesario crear programas de incentivo a los docentes para el uso de estas herramientas en sus diferentes actividades.

Así mismo, se considera necesario seguir con este tipo de estudios que relacione el uso de las herramientas tecnológicas como Google u otra que se genere en el mundo de las tecnologías digitales y su beneficio en el aprendizaje de las diversas asignaturas en las universidades particulares y nacionales, además en las instituciones educativas de formación básica. Y se apunte, a generalizar la implementación de las herramientas Google en todas las instituciones educativas.

\section{Referencias Bibliográficas}

Aching, Cesar. (2005). Matemáticas financieras para toma de decisiones empresariales. Ediciones Eumed.net. Perú. Extraído de: http://www.adizesca.com/site/assets/mematematicas financieras para toma de decisiones empresariales-ca.pdf Consulta: 29/10/19.

Avello, Raidell y Duart, Josep. (2016). Nuevas tendencias de aprendizaje colaborativo en elearning: Claves para su implementación efectiva. Estudios pedagógicos. Vol. 42 (1), Chile (Pp. 271-282).

Barrios, Irisysleyer y Casadei Carniel, Luisa (2014). Promoviendo el Uso de Google Drive como Herramienta de Trabajo Colaborativo en la Nube para Estudiantes de Ingeniería. Revista de Tecnología de Información y Comunicación en Educación. Vol. 8 (1), (Pp. 43-55). 
gle_Drive_como_Herramienta_de_Trabajo_Colaborativ_o_en_la_Nube_para_Estudi antes de Ingenieria/link/0046353c71ee1ab28e000000/download Consulta: 29/10/19.

Borras, Oriol y Pérez, Ana. (2017). Uso de Hangouts como recurso educativo en abierto en MOOC. IV Congreso Internacional sobre Aprendizaje, Innovación y Competitividad. Universidad Politécnica de Madrid, España.

Camargo Uribe, Ángela y Hederich Martínez, Christian (2010). Jerome bruner: dos teorías cognitivas, dos formas de significar, dos enfoques para la enseñanza de la ciencia. Psicogente, Vol.13 (24), 329-346. Extraído de: https://www.redalyc.org/articulo.oa?id=4975/497552357008 Consulta: 28/10/19.

Castro, Jeannette. (2007). La investigación en educación matemática: Una hipótesis de trabajo.

Educere. Investigación arbitrada. Mérida, Venezuela. Vol. 11 (38), (Pp. 519-531). Extraído de: http://ve.scielo.org/pdf/edu/v11n38/art19.pdf Consulta: 30/10/19.

Centorbi, Guillermo y Antonovich, Gastón (2017). Matemática financiera. Colegio Nacional Rafael Hernández. Universidad Nacional de la Plata. Extraído de: http://www.nacio.unlp.edu.ar/archivos/Optativas2017/6to/MatematicaFinanciera.pdf Consulta: 28/10/19.

Céspedes, John y Cossio, Giovanny. (2015). La enseñanza de los contenidos actitudinales de las ciencias Sociales: un análisis desde las prácticas docentes. Tesis Universidad de Antioquia. Colombia. Extraído de: http://ayura.udea.edu.co:8080/ispui/bitstream/123456789/2078/1/PB0944_Stiven_Gio vanny.pdf Consulta: 29/10/19.

Chávez, Julio y Villacorta, Paul. (2019). Influencia de la aplicación de herramientas de Google Drive en el desarrollo de competencias de aprendizaje colaborativo. Tesis de Grado. Maestro en Edumática y docencia universitaria. Universidad Tecnológica de Perú. Perú.

Díaz Barriga Arceo, Frida y Hernández Rojas, Gerardo (2010). Estrategias docentes para un aprendizaje significativo, Una interpretación constructivista. (2da. Ed.). McGraw Hill / Interamericana Editores. México.

Durán, José y Durán, Irene. (2018). TIC actualizadas para una nueva docencia universitaria. Mc Graw- Hill / Interamericana. España.

Galantini, José (2015). Plataforma Google Site como herramienta motivadora y la organización de recursos didácticos en estudiantes de maestría. Tesis de Grado. Maestro en Educación. Universidad de San Martin de Porres. Perú.

Garay, Victoria. (2012). Innovación educativa con TIC. Google docs, una herramienta para la construcción social del conocimiento en la FID. Revista Educación y Tecnología. $\mathrm{N}^{\circ}$ 1, Chile. (Pp 83-109).

González, Carlota, Veas, Alejandro, Fernández, Francisco, Jover, Irene Ignasi, Soriai, Priore, Américo Alejandro, González, Ma Milagros, García, Daniel (2018). Los procesos cognitivos de autorregulación clave para la mejora de la calidad en los diferentes aprendizajes en el ámbito universitario: la atención ejecutiva. Una experiencia en el aula. In Memorias del Programa de Redes-I3CE de calidad, innovación e investigación en docencia universitaria. (Pp. 847-864). Instituto de Ciencias de la Educación. España 
Hernández, Roberto, Fernández, Carlos y Baptista, Pilar. (2018). Metodología de la investigación. Mc Graw- Hill. México.

Iftakhar, Shampa. (2016). Google classroom: what works and how? Journal of Education and Social Sciences. Vol. 3 (1), (Pp.12-18). Extraído de: https://www.jesoc.com/wpcontent/uploads/2016/03/KC3 35.pdf Consulta: 25/10/19.

Leyva, Hermelinda, Vera, Monserrat y Pérez, Sandra. (2018). Google Forms en la evaluación diagnóstica como apoyo en las actividades docentes. Caso con estudiantes de la Licenciatura en Turismo. RIDE. Revista Iberoamericana para la Investigación y el Desarrollo Educativo. Vol. 9 (17), España. (Pp. 84-111). Doi: http://dx.doi.org/10.23913/ride.v9i17.374

Maldonado, Gilma (2014). Uso de las TIC como estrategia didáctica en el proceso de enseñanza de la geografía en $4^{\circ}, 5^{\circ}$ y $6^{\circ}$ grado de Educación Básica en la Escuela Normal Mixta Matilde Córdova de Suazo. Tesis de Grado. Maestro en Educación. Universidad Pedagógica Nacional Francisco Morazán República de Honduras.

Marmolejos Julio, Paulino, Edith y Gomez, R. (2014). Propuesta de estrategias que fomentan el aprendizaje y la solución de problemas en las ciencias básicas fortaleciendo la interpretación y aplicación del despeje, la sustitución numérica en ecuaciones y formulas, para los estudiantes del ciclo básico de la Universidad Autónoma de Santo Domingo. Congreso Iberoamericano de Ciencia, Tecnología, Innovación y Educación. Buenos Aires Argentina. Extraído de: https://www.oei.es/historico/congreso2014/memoriactei/449.pdf consulta: 28/10/19.

Montilva, Jonás, y Montilva, William. (2018). Un método ontológico-sistémico para el aprendizaje conceptual de tecnologías digitales. Ciencia e Ingeniería. Vol. 39 (3), Venezuela (Pp. 269-278).

Otero, Andrea, Rivera, Wilfred, Pedraza, Camilo y Canay, José. (2019). TIC para la educación: sistema adaptativo basado en mecanismos de aprendizaje automático para la apropiación de tecnologías en estudiantes de educación media. Revista Telos: Revista de Estudios Interdisciplinarios en Ciencias Sociales Vol. 21, No. 3. Venezuela (Pp. 526-543). Doi: www.doi.org/10.36390/telos213.03

Pérez Briceño, Juan Carlos, Iñiguez Ortega, Andrea, León Pineda, Sergio Mauricio. (2017) El aprendizaje de las matemáticas financieras. Analysis, Vol. 20 (2), (Pp. 1-13). DOI: 10.5281/zenodo. 1187129

Rojas, Alí. (2014). Didáctica Crítica de la Matemática Financiera Sapiens. Revista Universitaria de Investigación. Vol. 11, No. 2. Venezuela (Pp. 113-132).

Roque, Loida. (2017). Las TICS y su relación con el aprendizaje del área de Comunicación de los estudiantes del 5to año de la I.E. Augusto Salazar Bondy. Tesis de Grado. Maestro en Educación. Universidad Nacional Mayor de San Marcos. Perú.

Suárez Sánchez-Ocaña, Alejandro. (2012). Desnudando a Google: nacimiento de un imperio. Nueva Época. DERECOM. No. 10, (Pp. 99-115). Extraído de: https://dialnet.unirioja.es/servlet/articulo?codigo=4331531 Consulta: 25/10/19.

UNESCO. (2018). Informe presentado ante la cumbre iberoamericana para la educación de los países de América Latina y el Caribe Latina. Organización de las Naciones Unidas para la Educación, la Ciencia y la Cultura. España. 
Valdés, Michel. (2019). Internacionalización del currículo universitario virtual en el contexto de la globalización. Revista Telos: Revista de Estudios Interdisciplinarios en Ciencias Sociales. Vol. 21, No. 3. Venezuela (Pp. 754-775). Doi: www.doi.org/10.36390/telos 213.13

Valijas de herramientas TIC. (2016). Formularios de Google - Guía de uso. Extraído de: http://valijas.ceibal.edu.uy/storage/app/media/Guias\%20de\%20uso/Google\%20formul arios.pdf Consulta: 30/10/19.

Vélez, Mayra. (2016). Google Classroom en la enseñanza: Manual sobre las funciones básicas y mejores prácticas de uso. Departamento de Ciencia Política Universidad de Puerto Rico-Río Piedras, Puerto Rico. Extraído de: http://cea.uprrp.edu/wpcontent/uploads/2016/10/manual.pdf Consulta: 28/10/19. 